\title{
Radioactive isotopes in the interstellar medium
}

\author{
Roland Diehl ${ }^{1}$ (D)
}

Received: 1 April 2021 / Accepted: 9 October 2021 / Published online: 8 November 2021

(c) The Author(s) 2021

\begin{abstract}
Radioactive components of the interstellar medium provide an entirely-different and new aspect to the studies of the interstellar medium. Injected from sources of nucleosynthesis, unstable nuclei decay along their trajectories. Measurements can occur through characteristic gamma rays that are emitted with the decay, or in cosmic material samples through abundances of parent and daughter isotopes as they change with decay. The dynamics and material flows within interstellar medium are thus accessible to measurement, making use of the intrinsic clock that radioactive decay provides. We describe how measurements of radioactive decay have obtained a break-through in studies of the interstellar medium, after first summarizing the characteristics of radioactivity and the sources of unstable nuclei.
\end{abstract}

Keywords Interstellar medium · Gamma rays . Nucleosynthesis · Massive stars · Supernovae · Spectroscopy

\section{Introduction}

\subsection{Modern astronomy and radioactive nuclei}

A part of the gas in the interstellar medium is radioactive. Injected from sources of nucleosynthesis, radioactive newlyformed nuclei add special opportunities for studying the dynamic and complex interstellar medium. The radioactive decay imprints a clock into the abundance of these nuclei, as

This article belongs to the Topical Collection: Plasma, Particles, and Photons: ISM Physics Revisited. Guest Editors: Manami Sasaki, Ralf-Jürgen Dettmar, Julia Tjus.

$\varangle$ R. Diehl

1 Max-Planck-Institut für extraterrestrische Physik, Giessenbachstr. 1, 85741 Garching, Germany they decay from parent into stable daughter nuclei. The table of known isotopes holds about 40-50 radioactive isotopes with lifetimes that, on one hand, exceed the first 100 years where injection physics may dominate, and, on the other hand, are shorter than the age of the Galaxy. This makes such isotopes suitable, in principle, for a new type of study of the properties of the interstellar medium itself, using radioactivity as a property.

Astronomical measurements of radioactivity use two main methods: Characteristic nuclear emission lines measured with gamma-ray telescopes, and isotopic abundances in samples of cosmic matter captured within our solar system. Both of these methods are rather new and not-so familiar disciplines of astronomy, as illustrated in Fig. 1.

Radioactive decay of an unstable isotope is often accompanied by emission of characteristic radiation, gamma-ray line emission with energies that are specific to the nuclear levels of the daughter nucleus. Figure 2 shows the nuclear level and decay scheme for one example, the ${ }^{26} \mathrm{Al}$ isotope. This isotope has a lifetime of 1.04 Myrs, and decays mostly by $\beta^{+}$-decay into an excited state of ${ }^{26} \mathrm{Mg}$, which emits its excitation energy through characteristic gamma-ray line emission with an energy of $1808.63 \mathrm{keV} .{ }^{26} \mathrm{Al}$ was the first unstable isotope which was detected to decay in the interstellar medium (Mahoney et al. 1982). Its lifetime is shorter than typical times of stellar evolution, and much shorter than the age of the Galaxy itself or older stars herein. So it must have been produced recently and ejected from young, massive, and shortlived stars and possibly their terminal supernovae (see Prantzos and Diehl 1996; Diehl et al. 2021, for reviews).

Cosmic material had been the seed for all bodies of our solar system, including Earth, Moon, and meteorites. Today, cosmic dust and nuclei of high-energy cosmic rays reach instruments that have been launched into near-earth space. 


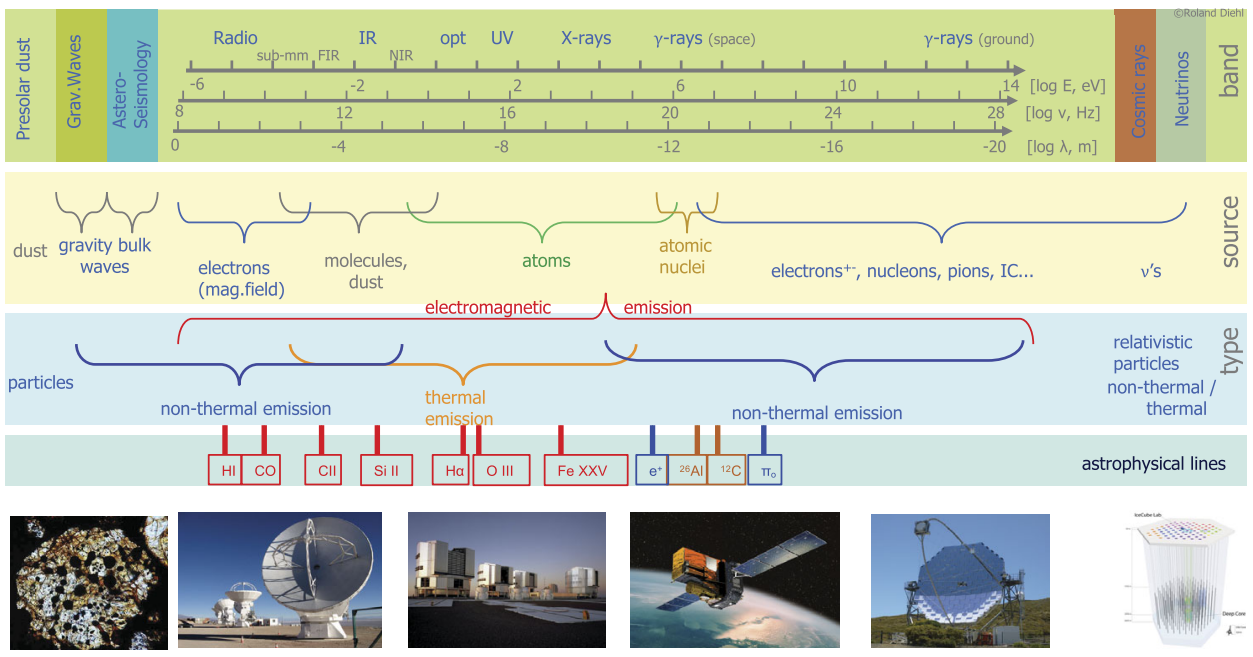

Fig. 1 The electromagnetic spectrum, from radio wavelengths to gamma-rays, covers more than 20 orders of magnitude, and includes signatures of different physical processes, as labelled. Specialised telescopes address mostly narrow astronomical windows, some from ground (optical, radio), and many on space platforms that have boosted

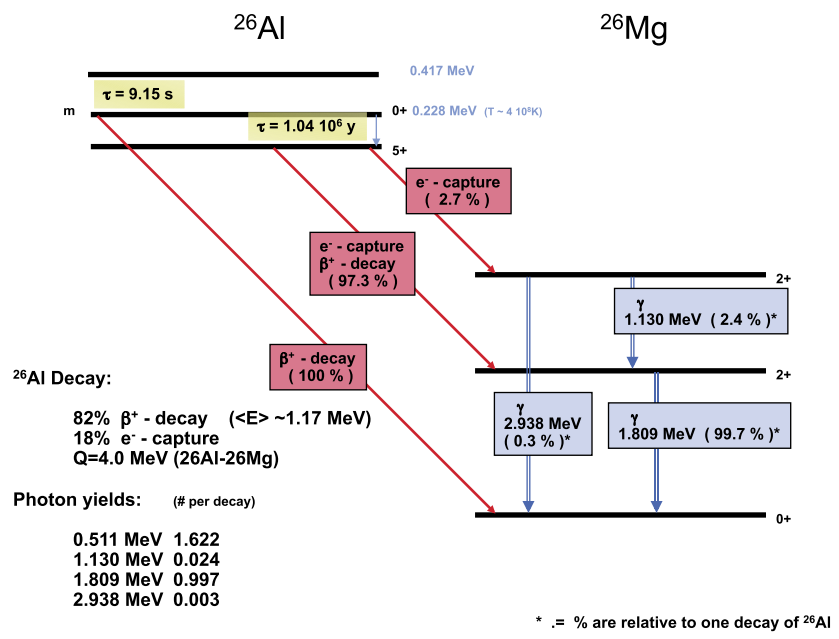

Fig. 2 Radioactivity is illustrated through the nuclear level and decay scheme of ${ }^{26} \mathrm{Al}$ decay (simplified): Gamma rays are emitted upon deexcitation of nuclear states in the daughter nucleus. For decay of ${ }^{26} \mathrm{Al}$, this includes gamma-rays from annihilation of the positrons produced in $\beta^{+}$decay

Analysing cosmic-material probes in terrestrial laboratories has provided rich detail about the full variety of isotopes and their abundances, both for cosmic rays and for dust particles in their great variety of mineral phases (Zinner 2008). Cosmic gas and dust that reaches our planet Earth from cosmic material flows may end up in sediments on Earth and moon. These minute admixtures of cosmic origins only could be found in a very sensitive method called accelerator mass spectrometry (Kutschera and Paul 1990). Other methods to analyse samples of cosmic materials in- the broad coverage of the different domains of the electromagnetic spectrum. Non-radiation based astronomies have been developed and complement these, such as measuring neutrinos and gravitational waves, and dust particles captured within the solar system by space satellites and on Earth and Moon

clude the ion-beam sputtering of meteoritic samples within secondary-ion mass spectrometry, NanoSIMS instruments (Hoppe et al. 2004) to determine precision isotopic abundances in small presolar grains, and thus measure characteristic daughters from radioactive decays. Through experimental efforts such as these, one may, therefore, consider the analysis of samples of cosmic materials also as an astronomy, though providing information about astrophysical processes and sources in different ways. Clearly, these methods are subject to a fundamental constraint of how the material sample was obtained, or formed in the first place. Upon closer examination, we note that also electromagnetic observations that have been used for species abundance determinations involve constraints of this type; e.g., a species must be radiation-coupled, thus in case of atomic line emission be not fully-ionised and excited by, e.g., collisions.

\subsection{Radioactivity and astrophysical studies}

The release of nuclear binding energy, as in radioactive decay, plays a prominent role for the appearance of many sources in astronomy throughout the universe. Stars shine, because nuclear reactions in the inner regions fuse lighter to heavier nuclei, which are more-tightly bound (Eddington 1919). Successive burning stages during the evolution of a star correspond to different nuclear fuels, each of which temporarily slows down the gravitational collapse of the star (Fig. 5). In supernova explosions, large amounts of radioactive ${ }^{56} \mathrm{Ni}$ are produced: Of order $0.1 \mathrm{M}_{\odot}$ are typically produced in core-collapse supernovae, as 
we learned from SN1987A $\left(0.07 \mathrm{M}_{\odot}\right)$ (Arnett et al. 1989; Fransson and Kozma 2002; McCray and Fransson 2016). SN1987A was the first core-collapse supernova where we were able to see gamma rays directly attributed to the radioactive energy input from ${ }^{56} \mathrm{Ni}$ decay: The Solar Maximum Mission and its Gamma-Ray Spectrometer instrument showed the characteristic lines from decay of ${ }^{56} \mathrm{Co}$ at 847 and $1238 \mathrm{keV}$, respectively (Matz et al. 1988). Thermonuclear supernovae (type Ia) produce even more ${ }^{56} \mathrm{Ni}$, typically $0.5 \mathrm{M}_{\odot}$, as we learned from many distant events (Scalzo et al. 2014; Seitenzahl and Townsley 2017), and saw with independent methods from SN2014J (Churazov et al. 2014; Diehl et al. 2015). The gamma-rays and positrons emitted from radioactive decay of ${ }^{56} \mathrm{Ni}$ energise the ejecta and envelope of the supernova from inside, and the scattered and re-processed radioactive energy is thus responsible for the light that appears at the photosphere and makes supernovae to be bright sources. Although this photospheric emission is key to many astrophysical applications, the radioactivity also directly provides a diagnostic of the explosions themselves. The radioactive energy injection of ${ }^{56} \mathrm{Ni}$ decay to power light from thermonuclear supernovae could be measured directly for the first time with INTEGRAL's spectrometer SPI (Diehl et al. 2014, 2015; Churazov et al. 2014). This is a valuable complement, as it bypasses the uncertainties and complexities of radiation transport within the supernova, that downscatters $\mathrm{MeV}$ radiation by many orders of magnitude into optical light. Its diagnostic power had been emphasised by modeling work (e.g. Summa et al. 2013) long before SN2014J occurred. The radioactive afterglow of the core-collapse supernova remnant Cas A in ${ }^{44} \mathrm{Ti}$ line emissions (Grefenstette et al. 2014) showed us the morphology of young ejecta, as they still are forming a remnant that also is bright in X-rays and radio emissions (Fig. 4). ${ }^{44} \mathrm{Ti}$ has a radioactive lifetime of 89 years, and thus had been helpful to study this remnant with about 350 years of age (Vink et al. 2000; Vink 2012). Also fluorescent X-ray line emission at $\mathrm{keV}$ energies may be emitted from radioactive material, if decay occurs through electron capture and leads to such $\mathrm{X}$-ray emission as the atomic-shell vacancy is replenished (Seitenzahl et al. 2015); this may have added to the energisation of the late SN1987A light curve, but direct detections of such fluorescence lines are still ambiguous (Borkowski et al. 2010).

$\mathrm{X}$-ray and radio continuum emissions of supernova remnants fade within thousands of years. The later supernova remnant phases, before they dissolve and merge with ambient gas, can be studied, if surrounding gas is dense enough, so that emission re-brightens upon the interaction of the supernova shock with the surrounding interstellar gas; the reverse shock at this phase also illuminates the remnant itself from the inside.

Beyond, at a later stage, longer-lived radioisotopes among the supernova ejecta trace what happens as ejecta merge into

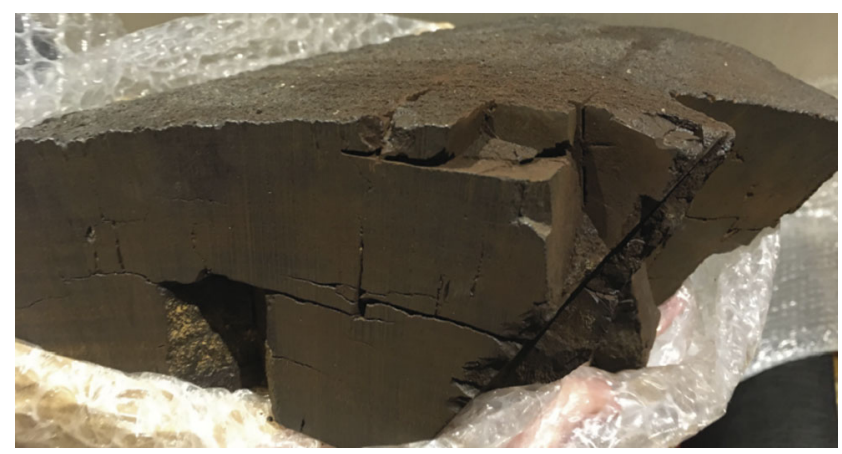

Fig. 3 This sample of a slowly-growing Manganese crust was retrieved from the deep pacific oceanfloor, remote from contaminations of debris of civilisation (photo courtesy Anton Wallner)

the ambient interstellar medium; this will be discussed in more detail in Sect. 3 below.

Radioactive decay is independent of the thermodynamic state and condition details of the gas. Therefore, the imprinted clock is independent of these, and is just a property of the specific nuclide. Thus, astronomical measurements of radioactive isotopes allow the study of propagation effects within the interstellar medium, using radioactivity tracing. ${ }^{1}$

Beyond studies in electromagnetic radiation, radioactivity has been exploited in samples of cosmic materials for astrophysical studies. Cosmic dust particles, called stardust, have been incorporated into meteorites that formed in the early solar system, and can be found therein. Their characteristic isotopic abundances can be exploited towards constraints on the formation sites of these dust particles. Grains attributed to AGB stars, but also to nova and supernova origins, have been discussed (see Zinner 1998; Clayton and Nittler 2004; Zinner 2008, for reviews). Moreover, cosmic rays captured with suitable instruments on space satellites from nearby interplanetary space have been found to include several radioactive isotopes, including ${ }^{14} \mathrm{C},{ }^{36} \mathrm{Cl},{ }^{26} \mathrm{Al}$, ${ }^{10} \mathrm{Be},{ }^{59} \mathrm{Ni}$, and others. Data from satellites (Israel et al. 2005, 2018), high-altitude balloons (Walsh et al. 2019), and the space station (Adriani et al. 2020) have been used to constrain the propagation, specifically the path lengths traversed, for cosmic rays, as their spallation reactions on heavier nuclei such as $\mathrm{Fe}$ have created these radioisotopes. For the case of ${ }^{59} \mathrm{Ni}$, its detection constrains the time between ejection from its nucleosynthesis source and the acceleration to cosmic-ray energies, because ${ }^{59} \mathrm{Ni}$ only decays through electron capture, and thus remains stable once fully ionised in cosmic rays after acceleration (Mewaldt et al. 2001; Wiedenbeck et al. 2001). Other dust and gas particles of cosmic origins also continuously reach our planet Earth and the moon, and are deposited in minute amounts as sediments. They had first been detected in a sample of

\footnotetext{
${ }^{1}$ We are familiar with measurements tracing radioactivity in medical applications
} 


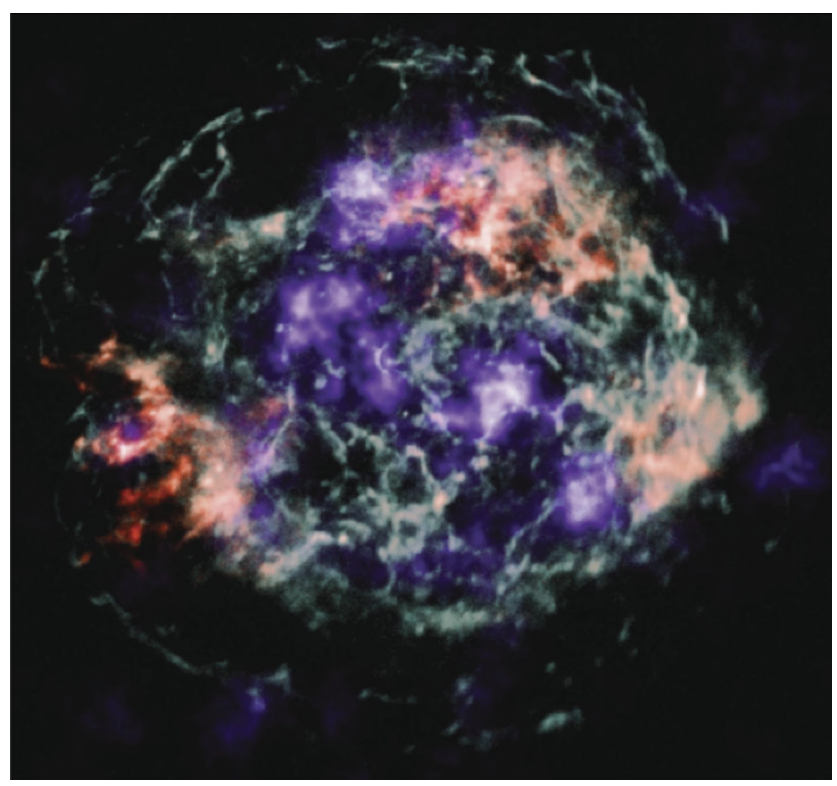

Fig. 4 The image of the Cas A supernova remnant demonstrates how radioactivity complements our view: Characteristic lines from ${ }^{44} \mathrm{Ti}$ decay (blue) reveal the location of inner ejecta, while X-ray line emissions from iron (red) and silicon (green) atoms, that also are emitted from those inner ejecta, show a somewhat different brightness distribution, due to ionization emphasising the parts of ejecta that have been shocked within the remnant. From Grefenstette et al. (2014)

an ocean crust from the deep pacific ocean floor, see Fig. 3, where slow sedimentation of materials has been found to include cosmic radioactivity in the form of ${ }^{60} \mathrm{Fe}$ isotopes (Knie et al. 1999; Wallner et al. 2016). More details and astrophysical lessons will be discussed in Sect. 3.2 below.

The context of this review is approached here from a viewpoint of nuclear gamma-ray spectroscopy. Nevertheless, we will also address other astronomical tools towards interstellar radioactivity, at least briefly. In this paper, we first present an overview of the different sources that are believed to be responsible for radioactive materials within the interstellar medium. Thereafter, we discuss the two main different astrophysical areas addressed by studies with radioactive tracers, (1) the transport of new nuclei from its sources towards mixing with the ambient interstellar gas, and (2) the use of radioactive tracers to learn about metallicity enrichment within galactic gas in general, within the cycle of matter including successive stellar generations.

\section{Sources of radioactive nuclei}

Radioactive substances are created by nuclear reactions mainly within stars and their explosions, and to some extent also within the interstellar medium. In stars, as densities and temperatures exceed the threshold for nuclear reactions within the stellar core. The trajectory in temperature and density for nuclear burning within massive stars is

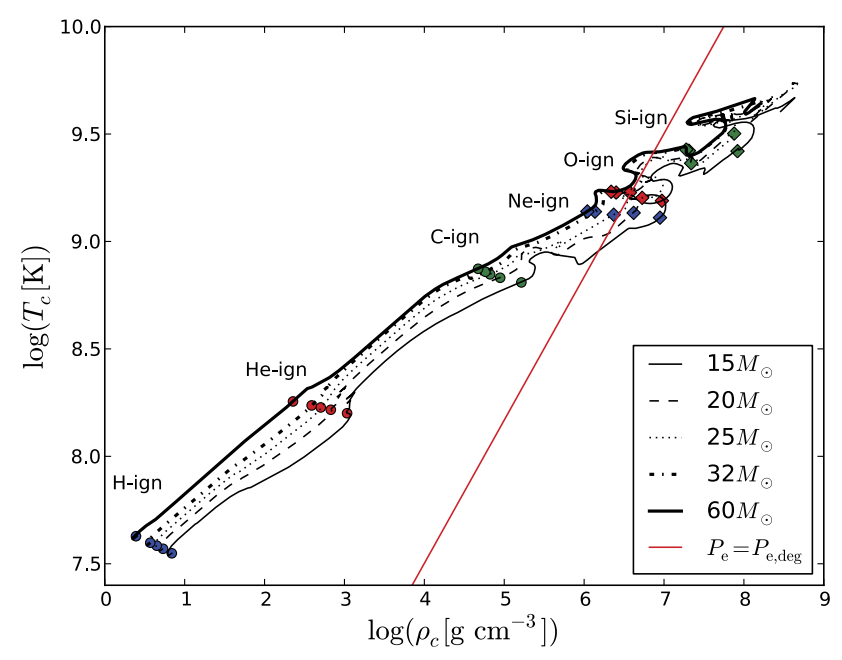

Fig. 5 The track of stellar evolution for massive stars, including the different nuclear-burning stages. Shown is central density versus central temperature, for different evolutionary-track calculations using the NUGRID software. The dashed line indicates the transition into degenerated gas in the core. From Pignatari et al. (2016)

shown in Fig. 5. Within stellar explosions, densities within the inner supernova approach values for nuclear matter, and temperatures exceed GK. Under such conditions, matter experiences what is called nuclear statistical equilibrium, and the nucleons available from the local composition of matter, i.e. protons and neutrons, mostly with a mild excess of the population of neutrons, is processed as guided by the binding energy of nuclei that may form in this region of phase space. For example, if the fuel consists of nuclei of equal proton and neutron numbers, such as ${ }^{4} \mathrm{He}$ or ${ }^{28} \mathrm{Si}$, the main product would be ${ }^{56} \mathrm{Ni}$. Generally, nuclear binding energy is maximised within the realm of iron-group nuclei. Such density and temperature are only realised in explosive environments, and possibly near highly compact and dynamic accretion disks that are in the process of forming a black hole. Thermonuclear supernova explosions that are launched from compact white dwarf stars at densities near $10^{10} \mathrm{~g} \mathrm{~cm}^{-3}$ clearly satisfy such conditions, and hence their main burning products are nuclei of the iron group, with a substantial amount of radioactive ${ }^{56} \mathrm{Ni}$ of about $0.5 \mathrm{M}_{\odot}$ (see Seitenzahl and Townsley 2017, for a review). Blackhole accretion disks will also heat up matter to nuclear burning temperatures at their outer radius, with corresponding nucleosynthesis towards equilibrium burning (Surman et al. 2006). It remains to be seen if parts of such matter could possibly be ejected, owing to the dynamical extremes that may occur as a compact star merges into the black hole, or explosion kicks of a preceding core-collapse supernova that first formed a neutron star before fallback material increased its mass beyond stability.

Within cosmic sources of nucleosynthesis, the particular nuclear reactions obey a complex network of reactions, 
that involve also unstable nuclei produced alongside, and reaction products vary per source type and environment. As Fig. 5 shows, we distinguish several main burning phases in stars from their main fuels, starting with hydrogen (the main phase of stellar evolution), then helium (the giant phase, followed by carbon, neon, oxygen, and silicon burning states. The latter burning stages are much shorter than the main and giant phases, because neutrinos are produced within such burnings, and these carry away energy that otherwise could stabilise the star, causing more-rapid contraction and fuel consumption to avoid collapse. Once the central composition of the star reaches iron group nuclei, no further energy release can be obtained from nuclear fusion reactions, and the star collapses. Depending on mass of the star, this can lead directly to formation of a compact remnant, which, in the case of a black hole, would bury all stellar matter forever. For stars less massive than about $25-30 \mathrm{M}_{\odot}$, however, morelikely is the inversion of the collapse into a supernova explosion: infalling matter bounces off the inner nuclear-density wall, and is energized by neutrinos produced as protons are converted to neutrons to avoid Coulomb repulsion and form dense neutron-star matter. Such a supernova explosion proceeds with the bounce shock wave propagating through the infalling envelope of the entire star, leading to some explosive nuclear burning. The explosion thus ejects all ashes produced from nuclear burnings outside the Fe core of the star, i.e. the burning phases shown in Fig. 5 and corresponding shell burnings.

Typically, several $\mathrm{M}_{\odot}$ of material are ejected from corecollapse supernova explosions, including typically amounts less than $0.1 \mathrm{M}_{\odot}$ in the form of radioactive nuclei. Much of the latter is ${ }^{56} \mathrm{Ni}$, which decays within 8 days to ${ }^{56} \mathrm{Co}$, which decays to stable ${ }^{56} \mathrm{Fe}$ within 111 days. More-longlived radioactive isotopes within core-collapse ejecta include small amounts of ${ }^{22} \mathrm{Na}$ and ${ }^{60} \mathrm{Co}$ with radioactive lifetimes of 3.8 and 7.6 years, respectively, and some $10^{-4} \mathrm{M}_{\odot}$ of ${ }^{44} \mathrm{Ti}$ with a radioactive lifetime of 89 years.

Other sources of nuclearly-processed materials exist, beyond the massive stars that have been described so far. Most prominently, supernova explosions of another type, thermonuclear supernovae called type Ia, are important sources of interstellar radioactivity. Here, a thermonuclear runaway explosion of a white-dwarf star occurs, and disrupts the entire star, ejecting typically $1-1.4 \mathrm{M}_{\odot}$ of gas into its surroundings (Seitenzahl and Townsley 2017). In these explosions, nuclear-statistical equilibrium conditions are achieved, now for the majority of the processed and ejected material. This makes these objects bright sources usable for cosmological studies, through the energy that is released as the typically $0.5 \mathrm{M}_{\odot}$ of radioactive ${ }^{56} \mathrm{Ni}$ decay, energising the still-dense exploding matter so that its photosphere is a bright source of UV-optical-infrared light. We believe that these supernovae are the result of mass transfer from a companion star onto the white dwarf in a binary system, thus driving the white dwarf towards Carbon ignition near the Chandrasekhar mass limit at around $1.4 \mathrm{M}_{\odot}$. The occurrence of a supernova of type Ia is typically delayed to occur at a time of order Gyr after its initial formation as a binary star system (Ruiter et al. 2011); this differs from massive-star activity, which is prompt, i.e., within few tens of Myrs after star formation. This also implies that typical source environments will be different: massive stars are embedded in stellar clusters and/or near molecular clouds, whereas type-Ia supernovae may typically occur in regions that have been depleted in gas from massive-star feedback, or else the binary system may have moved away from the dense star-formation site into empty regions during its long evolutionary time.

Other sources of nucleosynthesis are nova explosions, which occur rather frequently, at a rate of about $30 \mathrm{y}^{-1}$ within our Galaxy (Shafter 2017). These also result from mass transfer within a binary system, but only require accumulation of a hydrogen mass critical to ignition on the white dwarf star. This occurs in WD systems with mass transfer rates of order $10^{-9} \mathrm{M}_{\odot} \mathrm{y}^{-1}$, and ejects typically $10^{-7}$ to $10^{-4} \mathrm{M}_{\odot}$ of material into its surroundings (Jose and Hernanz 1998).

More exotic and rare contributors are believed to result from binary evolution leading to neutron stars, and their collisions after loss of orbital energy to gravitational radiation (Metzger 2017). This scenario spectacularly has been confirmed by observations in 2017 of the GW170817 event: a trigger from a gravitational-wave event and a coincident short gamma-ray burst stimulated a rich diversity of observations of this kilonova and its afterglows. Ejection of significant amounts of material is predicted, up to $10^{-2} \mathrm{M}_{\odot}$, that probably includes r-processed ejecta of heavy elements (Wu et al. 2016). While such rare nucleosynthesis events, which may also include a rare subtype of core-collapse supernovae called jet supernovae, may be important to understand the cosmic abundance evolution of rare species such as gold or uranium that are attributed to the $r$ process nucleosynthesis of elements heavier than $\mathrm{Fe}$, they are not considered important for the dynamics and evolution of the interstellar medium of typical galaxies itself, due to their scarcity (Belczynski et al. 2018).

Within the interstellar medium itself, nuclear reactions occur as energetic cosmic ray nuclei collide with nuclei of ambient gas (Ramaty et al. 1979; Tatischeff and Gabici 2018). This spallation nucleosynthesis produces characteristic unstable isotopes as abundantly-occurring nuclei of the interstellar medium such as carbon or iron are fragmented into lighter nuclei. The cosmic abundance of light elements $\mathrm{Li}-\mathrm{Be}-\mathrm{B}$ is understood to mainly result from such spallation in interstellar space, while contributions to other cosmic elemental abundances are minor (Prantzos 2010). The accretion-disk environments discussed above would provide 
conditions of such energetic particle collisions, particularly as the polar plasma jet of the newly forming black hole provides relativistic particles that can propagate over kpc distances, to eventually collide with colder interstellar gas clouds. Near active galactic nuclei, such a scenario has been used to predict nuclear de-excitation line fluxes (Mannheim 1997). More plausibly, in young supernova remnants the outward-moving shocks provide a setup that likely leads to particle acceleration and cosmic ray production. The cosmic rays that locally collide with ambient matter will thus incur de-excitation emission of nuclear origin, that should be measurable with next-generation gamma ray telescopes (Tibolla et al. 2011; Summa et al. 2011) (see Tatischeff and Gabici 2018, for a recent review). Regions of high massivestar density also should provide such a cosmic-ray acceleration scenario. In the nearby Orion region, a first report of de-excitation line detections from ${ }^{12} \mathrm{C}$ and ${ }^{16} \mathrm{O}$ with the COMPTEL instrument (Bloemen et al. 1997) could not be confirmed in later data re-analysis (Bloemen et al. 1999).

\section{Applications of radioactivity: astrophysical studies of the interstellar medium}

The presence of radioactive material within the interstellar medium is fortunate: (i) it traces current and recent nucleosynthesis, and (ii) it enables new types of investigations on the flow and the dynamics within the interstellar medium.

Unstable nuclei are particularly useful for the study of interstellar-medium astrophysics when their radioactive lifetimes exceed the complexities of the mass ejection from nucleosynthesis sources itself. Such complexities include processes that are partly specific to each type of source or even its particular surroundings, at least within the first few years to maybe decades. Examples are stellar winds or supernovae that interact with circumstellar matter that had been expelled before, such as superluminal supernovae. But also phenomena in the appearances of supernova remnants are driven to a large extent by the collision of the expanding explosion with ambient matter. Here we aim to address aspects of the interstellar medium itself.

We first discuss how radioactivity can be used to trace and study interstellar-medium properties more generally and on the larger (multi-) kpc scale in a galaxy. This includes complementing diagnostics of interstellar-medium parameters, and in particular the subject of compositional evolution of interstellar gas, otherwise known as the topic of chemical evolution. Thereafter, we discuss the local environments of the sources of radioactivity on a scale of typically $100 \mathrm{pc}$, and how the activity of those sources may affect the interstellar medium itself in their surroundings in turn; this process is called feedback, as it shapes the star formation process that may follow the injection of fresh material into the interstellar medium. We thus arrive at nearby special examples, the Orion and Scorpius-Centaurus regions, and the neighbourhood of our own solar system.

\subsection{Interstellar-medium characteristics at large}

Among radioactive isotopes that can be measured in interstellar space, the gamma-ray emitters ${ }^{26} \mathrm{Al}$ and ${ }^{60} \mathrm{Fe}$ are most important, as they have lifetimes of Myrs. Thus they can be observed long after newly-synthesised nuclei have been ejected into surroundings of sources. And, moreover, the typical nucleosynthesis injection rates (see Sect. 2) are high enough even in entire star-forming regions to not be dependent on few individual sources. Their emission arises from cumulative contributions of sources of stellar nucleosynthesis over their radioactive lifetime. Therefore, they provide a most-direct measure of such stellar nucleosynthesis activity on larger scales, from star-forming regions to the entire Galaxy.

Such a measurement is most-direct, as the emission arises from radioactive decay, which is the property of a nuclear species that is independent from environmental conditions that would be characterised by thermodynamic parameters such as temperature, and also independent of density and of degrees of ionisation. By comparison, observables such as counting of stars in the optical, measuring the temperature of dust in the infrared, and counting supernova remnants in radio emission, are less directly related to sources of nucleosynthesis, also because occultation, dust presence and size distributions, and ambient-medium densities, respectively, lead to systematic distortions that are difficult to assess, for a proper interpretation of measurements.

${ }^{26} \mathrm{Al}$ decays within $1.04 \mathrm{My}$, while ${ }^{60} \mathrm{Fe}$ is somewhat longer-lived, with a radioactive lifetime of $3.4 \mathrm{My}$. Both these long-lived isotopes are produced inside massive stars, however at different times and in different regions inside the star (see Fig. 6). ${ }^{26} \mathrm{Al}$ decay is directly observed in interstellar space through its characteristic $\gamma$ rays, which have an energy of $1808.65 \mathrm{keV}$. The pioneering observation of its existence and decay within the interstellar medium was made with the HEAO-C satellite of the US NASA space science program, as a proof of currently-ongoing nucleosynthesis in our Galaxy (Mahoney et al. 1982). The COMPTEL sky survey, made with the Compton Gamma-ray Observatory satellite (1991-2000), provided a first all-sky image in the ${ }^{26} \mathrm{Al} \gamma$-ray line (Diehl et al. 1995). The image showed structured ${ }^{26} \mathrm{Al}$ emission, extended along the plane of the Galaxy (Plüschke et al. 2001; Diehl et al. 1995) (see Fig. 7). This is in broad agreement with earlier expectations of ${ }^{26} \mathrm{Al}$ being produced throughout the Galaxy, and mostly from massive stars and their supernovae. The similarity in spatial distribution of this emission and the inferred 


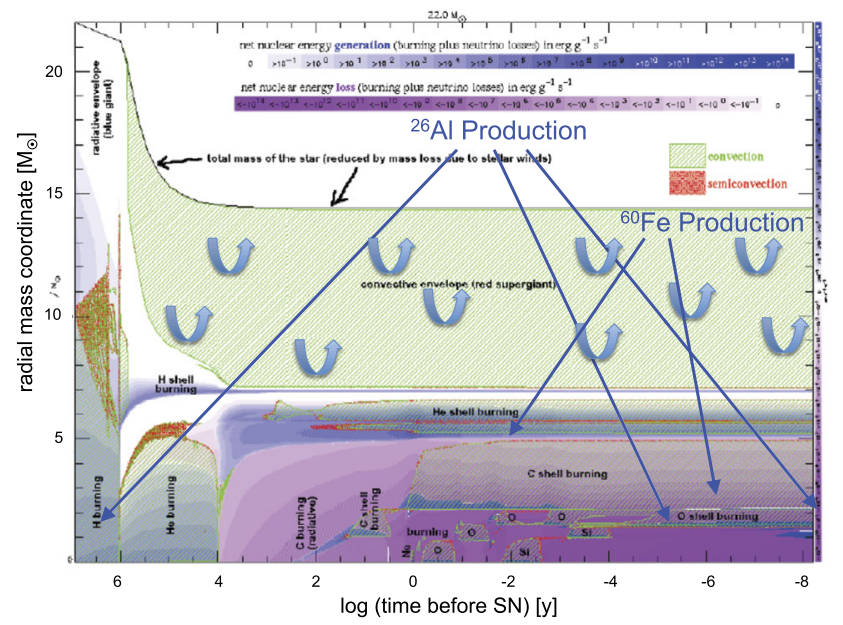

Fig. 6 The radioactive isotopes and gamma-ray emitters ${ }^{26} \mathrm{Al}$ and ${ }^{60} \mathrm{Fe}$ are both produced in massive stars, albeit in different regions of the star and at different times during evolution. In the representation of a massive star's evolution of this graph (Kippenhahn diagram, courtesy A. Heger) the production regions are indicated. While hydrostatic ${ }^{26} \mathrm{Al}$ production early on may end up in the surface mass lost from wind, all later and more-inside productions can only be ejected with the supernova

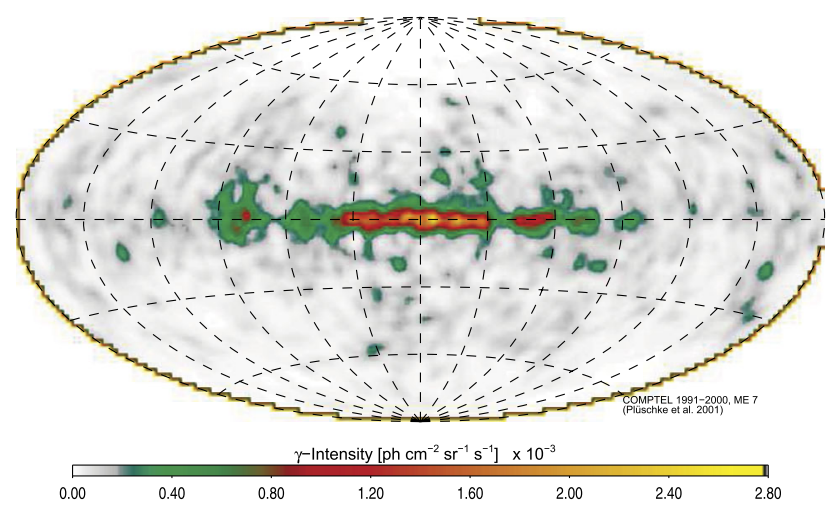

Fig. 7 The ${ }^{26} \mathrm{Al}$ sky as imaged with data from the COMPTEL telescope on NASA's Compton Gamma-Ray Observatory. This image (Plüschke et al. 2001) was obtained from measurements taken 1991-2000, and using a maximum-entropy regularization together with likelihood to iteratively fit a best image to the measured photons

locations of massive and young stars within the Milky Way Galaxy led to the interpretation that these were the dominating sources of ${ }^{26} \mathrm{Al}$ (Prantzos and Diehl 1996), although novae and AGB stars also had been advertised as promising contributors. It is not always straightforward, though, to interpret measurements of $\gamma$-ray line emission. The inherent blurring of the measurement by the response function of the $\gamma$-ray telescopes, and their inherently-high background, require sophisticated deconvolution and fitting methods to obtain the result shown in Fig. 7; remaining noise is evident. Bayesian methods need to be applied, and simple methods of subtracting background and inverting data with the instrument response matrix are not feasible. Nevertheless, the

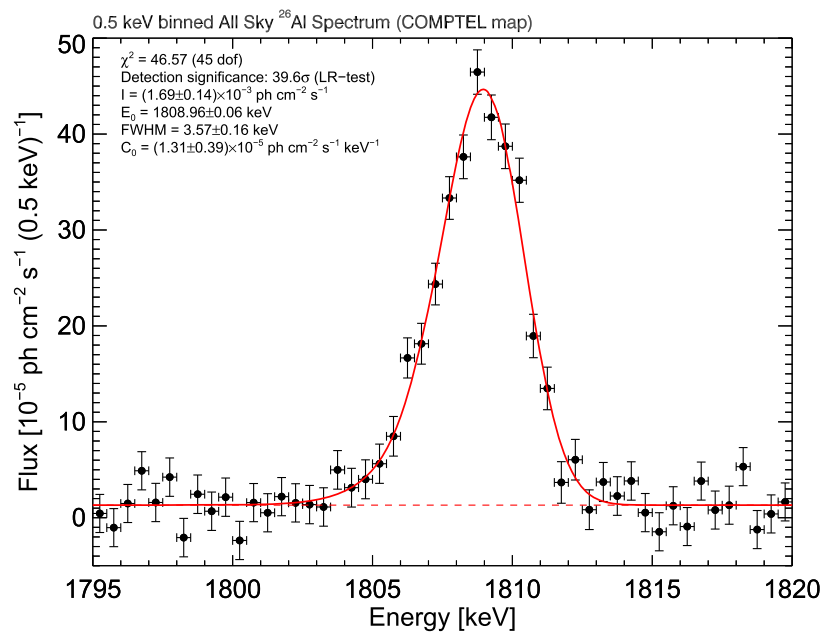

Fig. 8 The ${ }^{26} \mathrm{Al}$ line as seen with INTEGRAL's high-resolution spectrometer SPI and 13 years of measurements integrated (Siegert 2017)

sensitivity of current $\gamma$-ray surveys penetrates to intensities as low as few $10^{-6} \mathrm{ph} \mathrm{cm}^{-1} \mathrm{~s}^{-1}$. Typical exposure times are of durations of at least one month and longer.

A quantitative interpretation of the measured flux of ${ }^{26} \mathrm{Al}$ emission in terms of stellar nucleosynthesis activity is, however, still dependent on a fundamental difficulty in astronomical observation: Apparent brightness of a source on the sky needs to be related to the distance (and age, to address evolution) of the source. The distance of sources cannot be determined from the measured gamma rays, it has to be inferred from the observed morphology of the emission, using plausible assumptions. In Fig. 7, we see that the plane of the Galaxy appears, with a brighter ridge of emission in the directions towards the central regions of the Galaxy.

A second, supporting argument that the emission observed from ${ }^{26} \mathrm{Al}$ is of large-scale, galactic, origins is derived from the observed signature of large-scale Galactic rotation. ESA's INTEGRAL space observatory, launched in 2002 and expected to operate till 2029, carries a Ge-detector based spectrometer called SPI. This instrument is suitable for high-quality spectroscopic measurements, with a resolution of $3 \mathrm{keV}$ at the energy of the ${ }^{26} \mathrm{Al}$ line $(1809 \mathrm{keV})$. This spectral resolution corresponds to a Doppler velocity shift resolution of about $100 \mathrm{~km} \mathrm{~s}^{-1}$ for bright source regions (Kretschmer et al. 2013). COMPTEL was a firstgeneration gamma-ray telescope; it's scintillation detectors, however, lacked the spectral resolution required, for example, for line identification and spectroscopic studies towards dynamics of the interstellar ${ }^{26} \mathrm{Al}$ : it had $\simeq 200 \mathrm{keV}$ instrumental resolution, compared to $\simeq 3 \mathrm{keV}$ for Ge detectors, at the energy of the ${ }^{26} \mathrm{Al}$ line. This had been decisive for the pioneering HEAO-C measurement to convince scientists that they had indeed detected the line from the decay of the ${ }^{26} \mathrm{Al}$ isotope, rather than any other nuclide. A 1995 balloon experiment (named GRIS) that carried high-resolution $\mathrm{Ge}$ 


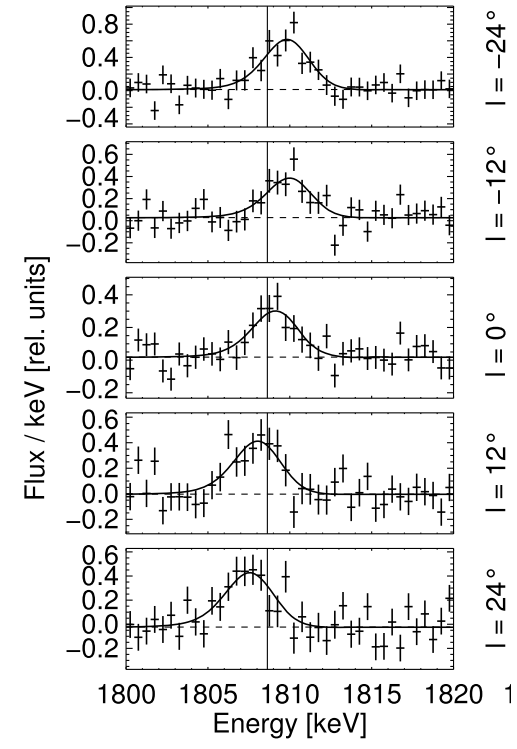

Fig. 9 The ${ }^{26} \mathrm{Al}$ line as seen towards different directions (in Galactic longitude) with INTEGRAL's high-resolution spectrometer SPI. This demonstrates kinematic line shifts from the Doppler effect, due to large-scale Galactic rotation (Kretschmer et al. 2013)

detectors had provided an indication that the ${ }^{26} \mathrm{Al}$ line was significantly broadened to $6.4 \mathrm{keV}$ (Naya et al. 1996). The quest was out to exploit this new capability in terms of astrophysical processes. Already in early results, the characteristic signature of large-scale Galactic rotation was clearly indicated (Diehl et al. 2006), i.e., a blue shift when viewing towards the fourth quadrant (objects on Galactic orbits approaching) and a red shift when viewing towards the first quadrant (receding objects, on average). With more exposure, this signature of large-scale Galactic rotation could be consolidated, as shown in Figs. 9 and 10. Thus, the observed gamma-ray flux could be translated into an observed total emitting mass of ${ }^{26} \mathrm{Al}$, making use of geometrical models of how sources are distributed within the Galaxy, such as double-exponential disks and spiral-arm models (see Diehl et al. 2006, for details). The original result of $2.8 \pm 0.8 \mathrm{M}_{\odot}$ (Diehl et al. 2006) was later revised and refined, as better geometrical models could be developed to account for foreground emission, and now suggests a somewhat lower total mass of $\mathrm{Al}$ in the Galaxy between $1.8 \mathrm{M}_{\odot}$ and the earlier value of $2.8 \mathrm{M}_{\odot}$ (Pleintinger 2020).

This observed mass of a single unstable isotope in the current Galaxy's interstellar medium can be converted into a rate of supernovae from massive stars (i.e., core-collapse supernovae). Supernovae are key to driving turbulence within the interstellar medium (Krumholz et al. 2018; Koo et al. 2020), hence their rate is a key parameter to understand the dynamical state of interstellar medium. Using ${ }^{26} \mathrm{Al}$ yields from theoretical models of massive stars and their supernovae, Diehl et al. (2006) derived a core-collapse supernova rate of $1.9 \pm 1.1$ events per century for our Galaxy; this

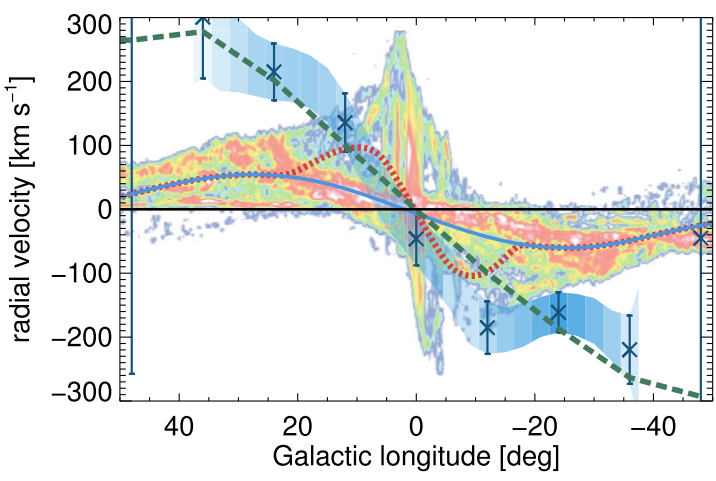

Fig. 10 The line-of-sight velocity shifts seen in the ${ }^{26} \mathrm{Al}$ line versus Galactic longitude, compared to measurements for molecular gas (Kretschmer et al. 2013)

value was updated with better ${ }^{26} \mathrm{Al}$ mass measurements (see above) and model yields to $1.4 \pm 1.1$ century $^{-1}$ (Diehl et al. 2018; Pleintinger 2020). This is quivalent to one such supernova in our Galaxy occurring every 71 years. At face value, the 294 supernova remnants observed in the Galaxy (Green 2019) thus present a tension with this value, as they would suggest a maximum sampling age of $21000 \mathrm{y}$, while significantly larger ages have been inferred for some of these. This, however, may be just another illustration of the dependence of the supernova remnant appearance on their surroundings.

The comparison of the observed velocity signature from large-scale Galactic rotation for ${ }^{26} \mathrm{Al}$ emission (Kretschmer et al. 2013) with the velocity signatures of other objects that orbit in such large-scale Galactic motion, however, lead to another puzzle (Fig. 10). The underlying question already had been opened by the GRIS measurement (Naya et al. 1996): if interpreted as kinematic Doppler broadening of astrophysical origin, translates into an ${ }^{26} \mathrm{Al}$ motion of $540 \mathrm{~km} \mathrm{~s}^{-1}$. Considering the $1.04 \times 10^{6} \mathrm{y}$ decay time of ${ }^{26} \mathrm{Al}$, such a large velocity observed for averaged interstellar decay of ${ }^{26} \mathrm{Al}$ would naively translate into kpc-sized cavities around ${ }^{26} \mathrm{Al}$ sources, so that velocities at the time of ejection would be maintained during the radioactive lifetime. An alternative hypothesis was that major fractions of ${ }^{26} \mathrm{Al}$ would be condensed on grains, which would maintain ballistic trajectories in tenuous interstellar medium (Chen et al. 1997; Sturner and Naya 1999).

The velocities seen for ${ }^{26} \mathrm{Al}$ throughout the plane of the Galaxy with SPI on INTEGRAL by Diehl et al. (2006) and Kretschmer et al. (2013) exceed the Galactocentric rotation velocities measured for several other object classes, such as molecular clouds, stars, and maser sources, by typically as much as $200 \mathrm{~km} \mathrm{~s}^{-1}$ (see Fig. 10). This high apparent velocity seen for decaying ${ }^{26} \mathrm{Al}$ as averaged along the line of sight means that ${ }^{26} \mathrm{Al}$ velocities remain higher than the velocities within typical interstellar gas for $10^{6}$ years, at least in the direction of Galactic rotation. This has been interpreted as ${ }^{26} \mathrm{Al}$ decay occurring preferentially within large cavities 


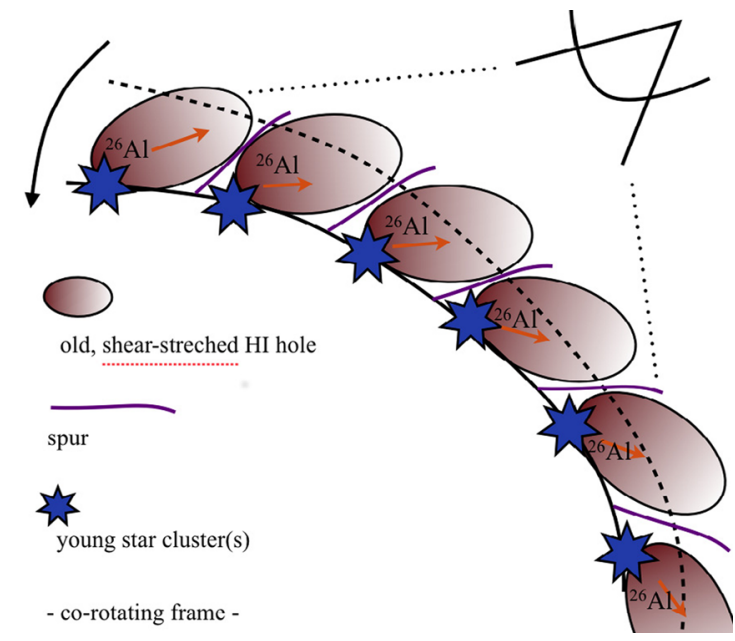

Fig. 11 A model for the different longitude-velocity signature of ${ }^{26} \mathrm{Al}$, assuming ${ }^{26} \mathrm{Al}$ blown into inter-arm cavities at the leading side of spiral arms (Krause et al. 2015)

(superbubbles), which are asymmetric and elongated away from sources into the direction of large-scale Galactic rotation (Fig. 11). If such cavities are interpreted as resulting from the early onset of massive-star winds in massivestar groups, wind-blown bubbles would arise and characterise the source surroundings at times when stellar evolution terminates in the core-collapse of most-massive stars. Such wind-blown superbubbles around massive-star groups plausibly extend further in space in lower-density directions forward and away from spiral arms (that host the sources). This has been seen in imaging studies of interstellar gas from other galaxies to occur (Schinnerer et al. 2019, and references therein). Superbubbles can have extensions up to kpc (Krause et al. 2015) (see also Rodgers-Lee et al. 2019; Krause et al. 2021), which allows ${ }^{26} \mathrm{Al}$ nuclei to propagate at speeds of the sound velocity within superbubbles of about $400 \mathrm{~km} \mathrm{~s}^{-1}$, and not being much decelerated until their decay and gamma-ray emission. One of the main open questions herein is the dynamics of superbubbles towards the Galactic halo above the disk; this is perpendicular to the line of sight, so that measurements of Doppler velocities cannot provide an answer.

The comparison of spatial distributions of candidate sources with the observed emission (Diehl et al. 1996) suggested that the ejection of ${ }^{26} \mathrm{Al}$ produced extended emission. The inferred galactic emission scale height of about 180 pc (see also Wang et al. 2009, for improved constraints from ${ }^{26} \mathrm{Al}$ observations) clearly exceeds the scale height of parental molecular gas $(50 \mathrm{pc})$, and is closer to the scale height inferred for OB associations in the nearby Galaxy (Maíz-Apellániz 2004). In first attempts to understand this aspect, simulations can be exploited: In 3D hydro-simulations that provide results within the Galactic plane which compare well with observations, the simulation results towards other directions should be credible as well. Recent years have provided computational power for large-scale simulations of this type (Fujimoto et al. 2018; Rodgers-Lee et al. 2019), dedicated to trace the fate of interstellar-medium evolution on a time scale of $\geq 10^{6}$ years for major parts of the entire Galaxy. These simulations show consistently that chimneys reach out of the Galactic disk and towards the halo in regions of intense nucleosynthesis activity. But, as a note of caution, Pleintinger et al. (2019) have pointed out that such simulations cannot be interpreted literally in terms of spatial aspects, because initial conditions adopted herein do not properly represent the aspect of observing the Galaxy from our vantage point in the solar system. Comparisons with expectations can be made in different ways. Biases in both the observations and the theoretical predictions require great care in drawing astrophysical conclusions (Pleintinger et al. 2019). In particular, theoretical predictions often need to make assumptions about our Galaxy and its morphology. These are particularly critical for the vicinity of the solar position, as nearby sources would appear as bright emission that may dominate the signal (Fujimoto et al. 2020). As shown by Pleintinger et al. (2019), generic characteristics of the interstellar medium morphology can well be extracted from comparisons of simulations to observations, but locations and extents of such chimneys require realism of the setup of simulations near the clusters of nucleosynthesis sources. Therefore, it is necessary to develop an understanding of how such clusters of sources act to shape their environments.

\subsection{Interstellar environments of nucleosynthesis sources}

The processes shaping interstellar environments of sources of nucleosynthesis are of interest, as they (i) determine the creation or operation of nucleosynthesis sources, and (ii) as they transport newly-produced nuclei from sources into the wider interstellar medium and towards places of interest (observers, newly-forming objects). Both these aspects involve processes with characteristic time scales that are long compared to individual source/environment interactions (here, $10^{5}$ years may be considered an upper bound, i.e., the oldest observable supernova remnant phenomena), and short compared to time scales of galactic evolution as a whole (here, $10^{8}$ years may be considered a lower bound, i.e., time scales of a galaxy collision, or of spiral-arm formation or dissolving times, or the duration of an orbit of galactic rotation about its centre).

The analysis of ${ }^{26} \mathrm{Al}$ spectra from specific regions hosting clusters of nucleosynthesis sources was extended towards multi-wavelength studies using the stellar census and information on atomic and molecular lines from radio data, as well as hot plasma from X-ray emission (Voss et al. 2009, 2010; Krause et al. 2013, 2014). Such studies have taught us 


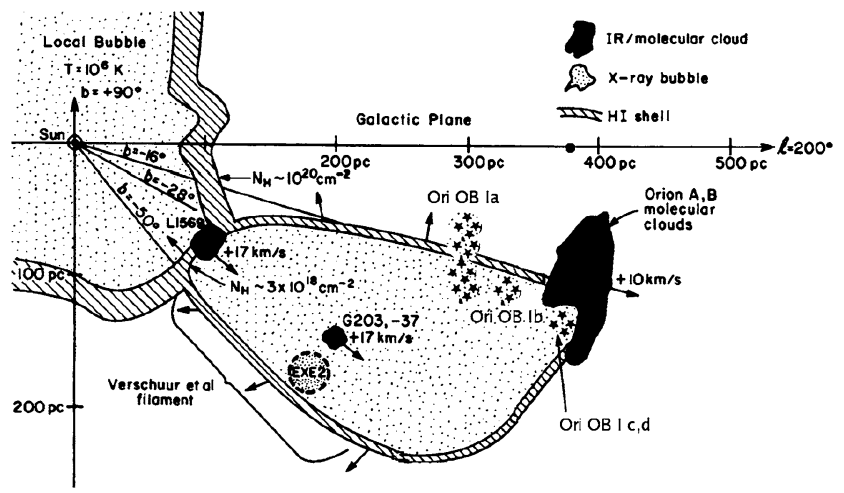

Fig. 12 The Orion region with the Eridanus cavity, illustrated from combined astronomical constraints (Fierlinger et al. 2012). This region at a distance of about $450 \mathrm{pc}$ for its massive stars of the Ori-Ob1 association and its subgroups forms an ideal laboratory to study superbubble astrophysics: The Eridanus cavity extends from the molecular clouds Orion A and B towards the Sun, and stars as well as gas structures are well mapped

that the ejection of new nuclei and their feeding into nextgeneration stars apparently is a complex process, less simple than the instantaneous recycling approximation used in many chemical evolution considerations. But being accessible to observational constraints such as from $\gamma$-ray line measurements shown for this case of ${ }^{26} \mathrm{Al}$, the ejection and transport of new nuclei can now be studied in more detail. ${ }^{26} \mathrm{Al}$ observations and their analyses have led to an ${ }^{26} \mathrm{Al}$ astronomy with studies of stellar feedback and massive-star nucleosynthesis (e.g., Krause et al. 2020).

The Orion region is the most-nearby example of massive star formation (Bally 2008). Star formation within the Orion A and B molecular clouds is understood to have created clusters of massive stars, the youngest now still observable as subgroups of the Orion OB1 association. Figure 12 shows the geometry of this region, collected from various literature by Fierlinger et al. (2012), as seen from the Sun at a distance of about $450 \mathrm{pc}$. Particularly interesting is the cavity called Eridanus, which extends from the Orion molecular clouds towards the Sun, and is delineated in observations from atomic hydrogen (HI) as the cavity walls show enhanced gas density, as well as in X rays from hot plasma as the cavity interior is heated by winds and supernovae from massive stars. Krause et al. (2014) have simulated a model for this situation of a cavity created by the energy (and mass) outputs from a cluster of stars. They show how overlapping of wind-blown bubbles leads to formation of a superbubble, and how later supernova explosions lead to periodic variations between excess heating and excess cooling in the interior of the cavity. The deep observations with INTEGRAL/SPI later were able to resolve the ${ }^{26} \mathrm{Al}$ decay gamma-ray line, and show an indication of the Doppler blueshift of the line, as ejecta from Orion OB1 stars expand into this cavity and towards the Sun (Fig. 13).

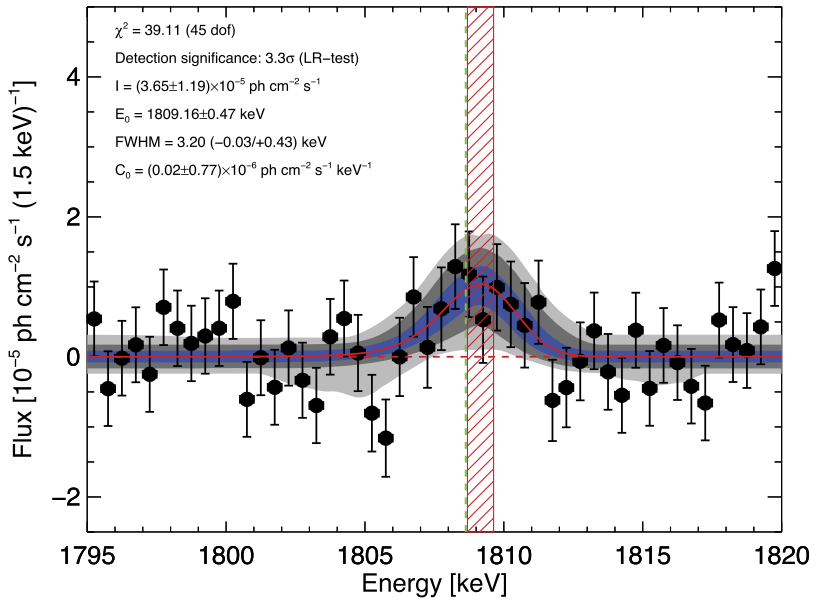

Fig. 13 The ${ }^{26} \mathrm{Al}$ line measurement from the Orion region indicates the blue shift that would be expected from the geometry (Fig. 12), with ejecta streaming towards us from the massive star groups located at the far side of the Eridanus cavity (Siegert 2017), although statistical precision is limited and deeper measurements are needed

Population synthesis had been employed as a tool to understand the collective action of a group of stars (Leitherer et al. 1999). In our implementation of such an accumulation of mass and energy ejections as extracted from stellar-evolution modeling results (Voss et al. 2009), we have shown how radioactive ejecta from ${ }^{26} \mathrm{Al}$ and ${ }^{60} \mathrm{Fe}$ can provide additional diagnostics of massive-star feedback. The Orion region matches such expectations, within the uncertainties of stellar census and subgroup ages, of energy estimated from the Eridanus cavity extents, and of observed radioactive emission (Voss et al. 2010).

As shown above (Fig. 7), there are many (at least several hundreds) of such massive-star cluster regions within the Galaxy. The Orion region has a favourable location for such studies, being sufficiently close for observational detail on stars and gas, yet being located at a distance away from the plane of the Galaxy, so that this region stands out from surrounding sky and can be distinguished from background at larger distances. Difficulties for cases of morenearby aspects have become evident, as the stellar groups of the Scorpius-Centaurus associations at only $140 \mathrm{pc}$ distance from the Sun have been studied (Preibisch et al. 2002; Pöppel et al. 2010). Nevertheless, also in this region, our general understanding of how massive stars form from molecular gas and erode their parental clouds through winds and supernovae is confirmed, when the current population of stars is compared against traces of past activity that pushes such gas and enriches it with radioactive nuclei (see detailed discussion in Krause et al. 2018). In this nearby example, one can recognise the star forming and nucleosynthesis activity over time, and may describe it as surround and squish action on parental molecular clouds, thus creating successive generations of massive star groups. This is reminiscent, but 
still significantly different from the triggered star formation scenario that (Preibisch et al. 2002) had proposed being exemplified by the Scorpius-Centaurus region's objects.

Our solar system has been found to also be enclosed within a major cavity, called the Local Bubble (Breitschwerdt et al. 1996). It is interesting to study the origins of this cavity, and to see if this can be related to the drivers of interstellar-medium turbulence, as discussed above (Lallement 2007). Radioactive admixtures to supernova ejecta again prove helpful in this context: The analysis of ocean crust samples from the deep and remote Pacific ocean floor had revealed inclusions of radioactive ${ }^{60} \mathrm{Fe}$ atoms (Knie et al. 1999, 2004). Through the extremelysensitive accelerator mass spectrometry (Kutschera and Paul 1990), which allows to find rare atomic nuclear species with a concentration as low as one in $10^{-15}, 69^{60} \mathrm{Fe}$ nuclei could be identified in the first such quantitative analysis (Knie et al. 1999). By now, other ocean crust and sediment samples across the surface of Earth, and also lunar samples, all have obtained the detections of such ashes attributed to core-collapse supernovae through the radioactive ${ }^{60} \mathrm{Fe}$ nucleus (Wallner et al. 2016). Importantly, the sedimentation process includes also records of other radioactive nuclei that can serve as a sedimentation clock, such as ${ }^{10} \mathrm{Be}$ produced in the Earth's atmosphere from cosmic-ray spallation. This isotope is used to determine ages of depths within the samples. It is now clear that the ${ }^{60} \mathrm{Fe}$ influx onto Earth is inhomogeneous in time, with interesting information. A clear peak of influx is found about 3 million years before present time, and a secondary peak is indicated near 8 Myrs (Wallner et al. 2016). To understand the astrophysical processes of nearby supernova explosions and ejecta propagation in turbulent and cavity-shaped interstellar medium, 3D studies of nearby objects have proven essential: It now appears that a nearby group of stars can be traced back in time to have been close to the Sun within the distance currently enclosed by the Local Bubble, and its supernovae have been creating this local bubble (Breitschwerdt and de Avillez 2006). Moreover, state-of-the-art 3D simulations used to trace the co-evolution of interstellar morphology and transport of supernova ejecta (Breitschwerdt et al. 2016; Schulreich et al. 2017) have shown that several supernovae originating from the same group could have shed their ejecta onto Earth. The peak of enhanced ${ }^{60} \mathrm{Fe}$ influx appears too broad in time to be plausibly consistent with a single supernova ejecta cloud sweeping over the solar system (Breitschwerdt et al. 2016). These same simulations leave as another possibility the cavity walls of the local bubble, as it accumulates ejecta produced within the cavity: The Sun came close to the cavity walls at a time around 3 Myrs ago, and may thus have faced enhanced influx of these rather recent supernova ejecta.

It is interesting in this context that recent analyses of cosmic-ray measurements with the CRIS instrument aboard the ACE satellite (Binns et al. 2016) have also revealed a signal from radioactive ${ }^{60} \mathrm{Fe}$. Unlike most other radioactive nuclei found in cosmic rays, ${ }^{60} \mathrm{Fe}$ at the detected amount cannot be produced in high-energy spallation reactions as cosmic rays hit interstellar gas. Therefore, this current-day influx of ${ }^{60} \mathrm{Fe}$ nuclei is yet another proof that supernova activity occurs within reach of cosmic-ray transport towards and into the solar system. Clearly, the ocean crust signal demonstrates a special enhancement in time, 3 to 8 Myrs ago; but the cosmic-ray measurement shows that even today the interstellar medium in the solar vicinity is shaped by supernova explosions. Binns et al. (2016) estimate a distance of the ${ }^{60} \mathrm{Fe}$ sources of up to $1 \mathrm{kpc}$, from modeling cosmicray transport with some approximations.

With the successful experiences in detection of radioactive traces of cosmic nucleosynthesis in terrestrial archives, there is another exciting result, now in the context to study the origins of heaviest elements above the iron group. These are attributed to the neutron capture processes of the $\mathrm{s}$ and $r$ processes (Burbidge et al. 1957). For a long time, corecollapse supernovae and their neutrino-driven winds have been considered the most-promising sources of r-process nuclei (Cowan et al. 1982; Thielemann et al. 1993). Galactic archeology, through determination of stellar surface abundances for stars of different ages (with $\mathrm{Fe}$ abundance as a proxy for age), had shown an observed buildup of abundances of metals such as $\mathrm{O}, \mathrm{Mg}$, and $\mathrm{Si}$, which were typical for supernova explosions from massive stars. But, using Eu as a proxy for r-process elements, it seemed that the early Galaxy enrichment with r-process elements occurred in a different way. In particular, the large fluctuations in time of early Eu abundances suggested that r-process element production was not dominated by a frequently-occurring source such as common supernovae. Rare but efficient alternative sources were studied and proposed, such as neutron star collisions and rare core-collapse supernovae with high characteristic magnetic fields. The detection of a kilonova that occurred coincident with the collision of two neutron stars that had been found in its characteristic gravitational-wave signal was a milestone for supporting such theoretical studies. Early enthusiasm led to claims that now the source of r-process elements had been found in such kilonovae. But closer inspection of expected rates and their evolution in time showed that also neutron star collisions cannot plausibly satisfy the constraints from galactic archeology as discussed above; we may face a superposition of contributions from more than one rare type of nucleosynthesis sources (Côté et al. 2019; Thielemann et al. 2020).

${ }^{244} \mathrm{Pu}$ has a radioactive lifetime of $1.2 \times 10^{8} \mathrm{y}$. A detection of ${ }^{244} \mathrm{Pu}$ on Earth thus would represent a record of r-process nucleosynthesis near the solar system, similar to what has been found with ${ }^{60} \mathrm{Fe}$ from core-collapse supernovae. The ocean crust samples therefore have also been 
searched for rare nuclei at higher masses, which could shed light on r-process origins (Wallner et al. 2015, 2021). Detecting several nuclei of ${ }^{244} \mathrm{Pu}$ in an analysis of a consistent set of ocean crust samples showing also ${ }^{60} \mathrm{Fe}$ led Wallner et al. (2021) to conclude that core-collapse supernovae of the frequently-occurring type cannot be responsible for r-process materials traced by ${ }^{244} \mathrm{Pu}$, as a much higher detection count would be expected. Of course, propagation of ejecta from a nucleosynthesis event into ocean floor sediments is a complex sequence of different astro-physical processes. But even accounting for all uncertainties involved, it is remarkable that detection of even individual and single radioactive nuclei in terrestrial material can provide astrophysical constraints on types of nucleosynthesis events.

\subsection{Conclusions}

The interstellar medium receives injections of turbulent energy and of matter from nucleosynthesis as massive star winds and supernova explosions occur. Within the nucleosynthesis products, radioactive isotopes are co-injected, and can be used to trace both the injection sources as well as the flow of ejecta materials. In the medium surrounding nucleosynthesis sources, short-lived radioisotopes such as ${ }^{44} \mathrm{Ti}$ decay and reveal conditions within the individual sources. The Cas A supernova remnant is the most-prominent example of such events, and shows through its radioactive decay gamma rays that ejecta propagate in major clumps, rather than in concentric shells, into the interstellar medium. More long-lived radioisotopes such as ${ }^{26} \mathrm{Al}$ and ${ }^{60} \mathrm{Fe}$ accumulate around sources, as their decay times are longer than the frequency of occurrence of source ejections in clusters of massive stars. Measuring the characteristic gamma rays from their decays have revealed that large cavities around such massive-star clusters are frequently found, and mediate the flow of nucleosynthesis ejecta before dissolving in the interstellar medium. This may determine how efficiently nextgeneration stars can capture ejecta from these sources, versus other source types such as thermonuclear supernovae or neutron star mergers. Terrestrial and lunar sediments have also shown traces of cosmic ${ }^{60} \mathrm{Fe}$, and thus witness nearby supernova activity in the vicinity of the solar system. Radioactive ${ }^{244} \mathrm{Pu}$ has been measured in the same sediments, and can be interpreted as a constraint on r-process nucleosynthesis happening within some rarer type of event than ${ }^{60}$ Fe ejecting core-collapse supernovae.

These examples underline the complementary capabilities of interstellar medium studies through radioactive materials, and prominently with cosmic gamma ray spectrometers. INTEGRAL currently is the only space mission that features a spectrometer for nuclear cosmic gamma rays. This mission is far beyond its originally-planned lifetime of 5 years, and may not last for too long; it will end its orbital lifetime in 2029. New missions towards these science goals and broader multi-messenger studies at up to two orders of magnitude better sensitivity have been proposed, such as the Astrogam mission (de Angelis et al. 2018). It remains to be seen if the astrophysical community can find budgets for such a mission, within the suite of exciting other bis projects of astronomy.

Acknowledgements This work was supported by the Max Planck Society, by DLR and ESA, and through EU COST Action 160117.

Funding Note Open Access funding enabled and organized by Projekt DEAL.

Publisher's Note Springer Nature remains neutral with regard to jurisdictional claims in published maps and institutional affiliations.

Open Access This article is licensed under a Creative Commons Attribution 4.0 International License, which permits use, sharing, adaptation, distribution and reproduction in any medium or format, as long as you give appropriate credit to the original author(s) and the source, provide a link to the Creative Commons licence, and indicate if changes were made. The images or other third party material in this article are included in the article's Creative Commons licence, unless indicated otherwise in a credit line to the material. If material is not included in the article's Creative Commons licence and your intended use is not permitted by statutory regulation or exceeds the permitted use, you will need to obtain permission directly from the copyright holder. To view a copy of this licence, visit http://creativecommons.org/licenses/by/4.0/.

\section{References}

Adriani, O., Akaike, Y., Asano, K., Asaoka, Y., Bagliesi, M.G., et al: Direct measurement of the cosmic-ray carbon and oxygen spectra from $10 \mathrm{GeV} / n$ to $2.2 \mathrm{TeV} / n$ with the Calorimetric Electron Telescope on the International Space Station. Phys. Rev. Lett. 125(25), 251102 (2020). https://doi.org/10.1103/ PhysRevLett.125.251102. arXiv:2012.10319

Arnett, W.D., Bahcall, J.N., Kirshner, R.P., Woosley, S.E.: Supernova 1987A. Annu. Rev. Astron. Astrophys. 27, 629-700 (1989). https://doi.org/10.1146/annurev.aa.27.090189.003213

Bally, J.: Overview of the Orion Complex. The Northern Sky ASP Monograph Publications, vol. 4, p. 459 (2008)

Belczynski, K., Bulik, T., Olejak, A., Chruslinska, M., Singh, N., Pol, N., Zdunik, L., O’Shaughnessy, R., McLaughlin, M., Lorimer, D., Korobkin, O., van den Heuvel, E.P.J., Davies, M.B., Holz, D.E.: Binary neutron star formation and the origin of GW170817. ArXiv e-prints (2018). arXiv:1812.10065

Binns, W.R., Israel, M.H., Christian, E.R., Cummings, A.C., de Nolfo, G.A., Lave, K.A., Leske, R.A., Mewaldt, R.A., Stone, E.C., von Rosenvinge, T.T., Wiedenbeck, M.E.: Observation of the ${ }^{60} \mathrm{Fe}$ nucleosynthesis-clock isotope in galactic cosmic rays. Science 352, 677-680 (2016). https://doi.org/10.1126/science.aad6004

Bloemen, H., Bykov, A.M., Diehl, R., Hermsen, W., van der Meulen, R., Schönfelder, V., Strong, A.W.: COMPTEL spectral study of the inner galaxy. In: Dermer, C.D., Strickman, M.S., Kurfess, J.D. (eds.) Proceedings of the Fourth Compton Symposium. American Institute of Physics Conference Series, vol. 410, pp. 1074-1078 (1997). https://doi.org/10.1063/1.54085

Bloemen, H., Morris, D., Knödlseder, J., Bennett, K., Diehl, R., Hermsen, W., Lichti, G., van der Meulen, R.D., Oberlack, U., Ryan, J., Schönfelder, V., Strong, A.W., de Vries, C., Winkler, C.: The revised COMPTEL orion results. Astrophys. J. Lett. 521, L137-L140 (1999). https://doi.org/10.1086/312185 
Borkowski, K.J., Reynolds, S.P., Green, D.A., Hwang, U., Petre, R., Krishnamurthy, K., Willett, R.: Radioactive scandium in the youngest galactic supernova remnant G1.9+0.3. Astrophys. J. Lett. 724, L161-L165 (2010). https://doi.org/10.1088/2041-8205/ 724/2/L161. arXiv: 1006.3552

Breitschwerdt, D., de Avillez, M.A.: The history and future of the Local and Loop I bubbles. Astron. Astrophys. 452, L1-L5 (2006). https://doi.org/10.1051/0004-6361:20064989

Breitschwerdt, D., Egger, R., Freyberg, M.J., Frisch, P.C., Vallerga, J.V.: The local bubble origin and evolution. Space Sci. Rev. 78, 183-198 (1996). https://doi.org/10.1007/BF00170805

Breitschwerdt, D., Feige, J., Schulreich, M.M., Avillez, M.A.D., Dettbarn, C., Fuchs, B.: The locations of recent supernovae near the Sun from modelling ${ }^{60} \mathrm{Fe}$ transport. Nature 532, 73-76 (2016). https://doi.org/10.1038/nature17424

Burbidge, E.M., Burbidge, G.R., Fowler, W.A., Hoyle, F.: Synthesis of the elements in stars. Rev. Mod. Phys. 29, 547-650 (1957). https:// doi.org/10.1103/RevModPhys.29.547

Chen, W., Diehl, R., Gehrels, N., Hartmann, D., Leising, M., Naya, J.E., Prantzos, N., Tueller, J., von Ballmoos, P.: Implications of the broad ${ }^{26} \mathrm{Al} 1809 \mathrm{keV}$ line observed by GRIS. In: Winkler, C., Courvoisier, T.J.L., Durouchoux, P. (eds.) The Transparent Universe. ESA Special Publication, vol. 382, p. 105 (1997)

Churazov, E., Sunyaev, R., Isern, J., Knödlseder, J., Jean, P., Lebrun, F., Chugai, N., Grebenev, S., Bravo, E., Sazonov, S., Renaud, M.: Cobalt-56 $\gamma$-ray emission lines from the type Ia supernova 2014J. Nature 512, 406 (2014)

Clayton, D.D., Nittler, L.R.: Astrophysics with presolar stardust. Annu. Rev. Astron. Astrophys. 42, 39-78 (2004). https://doi.org/10. 1146/annurev.astro.42.053102.134022

Côté, B., Eichler, M., Arcones, A., Hansen, C.J., Simonetti, P., Frebel, A., Fryer, C.L., Pignatari, M., Reichert, M., Belczynski, K., Matteucci, F.: Neutron star mergers might not be the only source of r-process elements in the Milky Way. Astrophys. J. 875(2), 106 (2019). https://doi.org/10.3847/1538-4357/ab10db. arXiv:1809. 03525

Cowan, J.J., Cameron, A.G.W., Truran, J.W.: The thermal runaway r-process. Astrophys. J. 252, 348-355 (1982). https://doi.org/10. $1086 / 159562$

de Angelis, A., Tatischeff, V., Grenier, I.A., McEnery, J., Mallamaci, M., et al. (E-Astrogam Collaboration): Science with eASTROGAM. A space mission for $\mathrm{MeV}-\mathrm{GeV}$ gamma-ray astrophysics. J. High Energy Astrophys. 19, 1-106 (2018). https://doi. org/10.1016/j.jheap.2018.07.001. arXiv:1711.01265

Diehl, R., Dupraz, C., Bennett, K., Bloemen, H., Hermsen, W., Knoedlseder, J., Lichti, G., Morris, D., Ryan, J., Schoenfelder, V., Steinle, H., Strong, A., Swanenburg, B., Varendorff, M., Winkler, C.: COMPTEL observations of Galactic ${ }^{26} \mathrm{Al}$ emission. Astron. Astrophys. 298, 445 (1995)

Diehl, R., Bennett, K., Dupraz, C., Knoedlseder, J., Lichti, G., Morris, D., Oberlack, U., Ryan, J., Schoenfelder, V., Strong, A., von Ballmoos, P., Winkler, C., Chen, W., Hartmann, D., Prantzos, N.: Modelling the $1.8 \mathrm{MeV}$ sky: tracers of massive star nucleosynthesis. Astron. Astrophys. Suppl. Ser. 120, 321-325 (1996)

Diehl, R., Halloin, H., Kretschmer, K., Lichti, G.G., Schönfelder, V., et al.: Radioactive ${ }^{26} \mathrm{Al}$ from massive stars in the Galaxy. Nature 439, 45-47 (2006). https://doi.org/10.1038/nature04364. arXiv: astro-ph/0601015

Diehl, R., Siegert, T., Hillebrandt, W., Grebenev, S.A., Greiner, J., et al.: Early ${ }^{56} \mathrm{Ni}$ decay gamma rays from SN2014J suggest an unusual explosion. Science 345, 1162 (2014)

Diehl, R., Siegert, T., Hillebrandt, W., Krause, M., Greiner, J., Maeda, K., Röpke, F.K., Sim, S.A., Wang, W., Zhang, X.: SN2014J gamma rays from the ${ }^{56} \mathrm{Ni}$ decay chain. Astron. Astrophys. 574, A72 (2015). https://doi.org/10.1051/0004-6361/201424991. arXiv:1409.5477
Diehl, R., Siegert, T., Greiner, J., Krause, M., Kretschmer, K., et al.: INTEGRAL/SPI $\gamma$-ray line spectroscopy. Response and background characteristics. Astron. Astrophys. 611, A12 (2018). https://doi. org/10.1051/0004-6361/201731815

Diehl, R., Krause, M.G.H., Kretschmer, K., Lang, M., Pleintinger, M.M.M., et al.: Steady-state nucleosynthesis throughout the Galaxy. New Astron. Rev. 92, 101608 (2021). https://doi.org/10. 1016/j.newar.2020.101608

Eddington, A.S.: The sources of stellar energy. Observatory 42, 371-376 (1919)

Fierlinger, K.M., Burkert, A., Diehl, R., Dobbs, C., Hartmann, D.H., Krause, M., Ntormousi, E., Voss, R.: Molecular cloud disruption and chemical enrichment of the ISM caused by massive star feedback. In: Capuzzo-Dolcetta, R., Limongi, M., Tornambè, A. (eds.) Advances in Computational Astrophysics: Methods, Tools, and Outcome. Astronomical Society of the Pacific Conference Series, vol. 453, p. 25 (2012)

Fransson, C., Kozma, C.: Radioactivities and nucleosynthesis in SN 1987A. New Astron. Rev. 46, 487-492 (2002). https://doi.org/10. 1016/S1387-6473(02)00188-4. arXiv:astro-ph/0112405

Fujimoto, Y., Krumholz, M.R., Tachibana, S.: Short-lived radioisotopes in meteorites from Galactic-scale correlated star formation. Mon. Not. R. Astron. Soc. 480(3), 4025-4039 (2018). https://doi. org/10.1093/mnras/sty2132. arXiv: 1802.08695

Fujimoto, Y., Krumholz, M.R., Si, I.: Distribution and kinematics of ${ }^{26} \mathrm{Al}$ in the Galactic disc. Mon. Not. R. Astron. Soc. 497(2), 2442-2454 (2020). https://doi.org/10.1093/mnras/ staa2125. arXiv:2006.03057

Green, D.A.: A revised catalogue of 294 Galactic supernova remnants. J. Astrophys. Astron. 40(4), 36 (2019). https://doi.org/10.1007/ s12036-019-9601-6. arXiv:1907.02638

Grefenstette, B.W., Harrison, F.A., Boggs, S.E., Reynolds, S.P., Fryer, C.L., et al.: Asymmetries in core-collapse supernovae from maps of radioactive ${ }^{44} \mathrm{Ti}$ in CassiopeiaA. Nature 506, 339 (2014)

Hoppe, P., Ott, U., Lugmair, G.W.: NanoSIMS, the new tool of choice: ${ }^{26} \mathrm{Al},{ }^{44} \mathrm{Ti},{ }^{49} \mathrm{~V},{ }^{53} \mathrm{Mn},{ }^{60} \mathrm{Fe}$, and more. New Astron. Rev. 48, 171-176 (2004). https://doi.org/10.1016/j.newar.2003.11.025

Israel, M.H., Binns, W.R., Cummings, A.C., Leske, R.A., Mewaldt, R.A., von Stone, E.C., Rosenvinge, T.T., Wiedenbeck, M.E.: Isotopic composition of cosmic rays: results from the Cosmic Ray Isotope Spectrometer on the ACE spacecraft. Nucl. Phys. A 758, 201-208 (2005). https://doi.org/10.1016/j.nuclphysa.2005. 05.038

Israel, M.H., Lave, K.A., Wiedenbeck, M.E., Binns, W.R., Christian, E.R., et al.: Elemental composition at the cosmic-ray source derived from the ACE-CRIS instrument. I. ${ }_{6} \mathrm{C}$ to ${ }_{28} \mathrm{Ni}$. Astrophys. J. 865(1), 69 (2018). https://doi.org/10.3847/1538-4357/aad867

Jose, J., Hernanz, M.: Nucleosynthesis in classical novae: CO versus ONe white dwarfs. Astrophys. J. 494, 680 (1998). https://doi.org/ 10.1086/305244. arXiv:astro-ph/9709153

Knie, K., Korschinek, G., Faestermann, T., Wallner, C., Scholten, J., Hillebrandt, W.: Indication for supernova produced ${ }^{60} \mathrm{Fe}$ activity on Earth. Phys. Rev. Lett. 83(1), 18-21 (1999). https://doi.org/10. 1103/PhysRevLett.83.18

Knie, K., Korschinek, G., Faestermann, T., Dorfi, E.A., Rugel, G., Wallner, A.: ${ }^{60} \mathrm{Fe}$ anomaly in a deep-sea manganese crust and implications for a nearby supernova source. Phys. Rev. Lett. 93(17), 171103 (2004). https://doi.org/10.1103/PhysRevLett.93.171103

Koo, B.C., Kim, C.G., Park, S., Ostriker, E.C.: Radiative supernova remnants and supernova feedback. Astrophys. J. 905(1), 35 (2020). https://doi.org/10.3847/1538-4357/abcle7. arXiv:2011. 06322

Krause, M., Fierlinger, K., Diehl, R., Burkert, A., Voss, R., Ziegler, U.: Feedback by massive stars and the emergence of superbubbles. I. Energy efficiency and Vishniac instabilities. Astron. Astrophys. 550, A49 (2013). https://doi.org/10.1051/0004-6361/201220060. arXiv:1207.7231 
Krause, M., Diehl, R., Böhringer, H., Freyberg, M., Lubos, D.: Feedback by massive stars and the emergence of superbubbles. II. Xray properties. Astron. Astrophys. 566, A94 (2014). https://doi. org/10.1051/0004-6361/201423871. arXiv:1405.0037

Krause, M.G.H., Diehl, R., Bagetakos, Y., Brinks, E., Burkert, A., Gerhard, O., Greiner, J., Kretschmer, K., Siegert, T.: ${ }^{26}$ Al kinematics: superbubbles following the spiral arms? Constraints from the statistics of star clusters and HI supershells. Astron. Astrophys. 578, A113 (2015). https://doi.org/10.1051/0004-6361/ 201525847. arXiv: 1504.03120

Krause, M.G.H., Burkert, A., Diehl, R., Fierlinger, K., Gaczkowski, B., Kroell, D., Ngoumou, J., Roccatagliata, V., Siegert, T., Preibisch, T.: Surround and Squash: the impact of superbubbles on the interstellar medium in Scorpius-Centaurus OB2. Astron. Astrophys. 619, A120 (2018). https://doi.org/10.1051/0004-6361/ 201732416. arXiv: 1808.04788

Krause, M.G.H., Offner, S.S.R., Charbonnel, C., Gieles, M., Klessen, R.S., Vázquez-Semadeni, E., Ballesteros-Paredes, J., Girichidis, P., Diederik Kruijssen, J.M., Ward, J.L., Zinnecker, H.: The physics of star cluster formation and evolution. Space Sci. Rev. 216(4), 64 (2020). https://doi.org/10.1007/s11214-020-00689-4. arXiv:2005.00801

Krause, M.G.H., Rodgers-Lee, D., Dale, J.E., Diehl, R., Kobayashi, C.: Galactic ${ }^{26} \mathrm{Al}$ traces metal loss through hot chimneys. Mon. Not. R. Astron. Soc. 501(1), 210-218 (2021). https://doi.org/10.1093/ mnras/staa3612. arXiv:2011.08615

Kretschmer, K., Diehl, R., Krause, M., Burkert, A., Fierlinger, K., Gerhard, O., Greiner, J., Wang, W.: Kinematics of massive star ejecta in the Milky Way as traced by ${ }^{26} \mathrm{Al}$. Astron. Astrophys. 559, A99 (2013). https://doi.org/10.1051/0004-6361/201322563. arXiv: 1309.4980

Krumholz, M.R., Burkhart, B., Forbes, J.C., Crocker, R.M.: A unified model for galactic discs: star formation, turbulence driving, and mass transport. Mon. Not. R. Astron. Soc. 477(2), 2716-2740 (2018). https://doi.org/10.1093/mnras/sty852. arXiv:1706.00106

Kutschera, W., Paul, M.: Accelerator mass spectrometry in nuclear physics and astrophysics. Annu. Rev. Nucl. Part. Sci. 40, 411-438 (1990). https://doi.org/10.1146/annurev.ns.40.120190.002211

Lallement, R.: The local interstellar medium: peculiar or not? Space Sci. Rev. 130, 341-353 (2007). https://doi.org/10.1007/s11214007-9178-2

Leitherer, C., Schaerer, D., Goldader, J.D., Delgado, R.M.G., Robert, C., Kune, D.F., de Mello, D.F., Devost, D., Heckman, T.M.: Starburst99: synthesis models for galaxies with active star formation. Astrophys. J. Suppl. Ser. 123(1), 3-40 (1999). https://doi.org/10. 1086/313233. arXiv:astro-ph/9902334

Mahoney, W.A., Ling, J.C., Jacobson, A.S., Lingenfelter, R.E.: Diffuse galactic gamma-ray line emission from nucleosynthetic $\mathrm{Fe}-$ 60, Al-26, and Na-22 - preliminary limits from HEAO 3. Astrophys. J. 262, 742 (1982). https://doi.org/10.1086/160469

Maíz-Apellániz, J.: Massive young clusters. In: Alfaro, E.J., Pérez, E., Franco, J. (eds.) How Does the Galaxy Work? Astrophys. Space Sci. Library, vol. 315, p. 231 (2004). https://doi.org/10.1007/14020-2620-X46. arXiv:astro-ph/0310162

Mannheim, K.: AGN models: high-energy emission. In: GiraudHeraud, Y., van Tran Thanh, J. (eds.) Very High Energy Phenomena in the Universe; Moriond Workshop, p. 17 (1997). arXiv: astro-ph/9703184

Matz, S.M., Share, G.H., Leising, M.D., Chupp, E.L., Vestrand, W.T.: Gamma-ray line emission from SN1987A. Nature 331, 416-418 (1988). https://doi.org/10.1038/331416a0

McCray, R., Fransson, C.: The remnant of supernova 1987A. Annu. Rev. Astron. Astrophys. 54, 19-52 (2016). https://doi.org/10. 1146/annurev-astro-082615-105405

Metzger, B.D.: Kilonovae. Living Rev. Relativ. 20, 3 (2017). https:// doi.org/10.1007/s41114-017-0006-z. arXiv:1610.09381
Mewaldt, R.A., Yanasak, N.E., Wiedenbeck, M.E., Davis Astron, J., Binns, W.R., Christian, E.R., Cummings, A.C., Hink, P.L., Leske, R.A., Niebur, S.M., Stone, E.C., Von Rosenvinge, T.T.: Radioactive clocks and cosmic-ray transport in the Galaxy. Space Sci. Rev. 99, 27-39 (2001)

Naya, J.E., Barthelmy, S.D., Bartlett, L.M., Gehrels, N., Leventhal, M., Parsons, A., Teegarden, B.J., Tueller, J.: Detection of highvelocity ${ }^{26} \mathrm{Al}$ towards the Galactic centre. Nature 384, 44-46 (1996). https://doi.org/10.1038/384044a0

Pignatari, M., Herwig, F., Hirschi, R., Bennett, M., Rockefeller, G., et al.: NuGrid stellar data set. I. Stellar yields from $\mathrm{H}$ to Bi for stars with metallicities $Z=0.02$ and $Z=0.01$. Astrophys. J. Suppl. Ser. 225, 24 (2016). https://doi.org/10.3847/0067-0049/225/2/24. arXiv:1307.6961

Pleintinger, M.M.M.: Star groups and their nucleosynthesis. PhD thesis, Technische Universität München (2020)

Pleintinger, M.M.M., Siegert, T., Diehl, R., Fujimoto, Y., Greiner, J., Krause, M.G.H., Krumholz, M.R.: Comparing simulated ${ }^{26} \mathrm{Al}$ maps to gamma-ray measurements. Astron. Astrophys. 632, A73 (2019). https://doi.org/10.1051/0004-6361/201935911. arXiv: 1910.06112

Plüschke, S., Diehl, R., Schönfelder, V., Bloemen, H., Hermsen, W., Bennett, K., Winkler, C., McConnell, M., Ryan, J., Oberlack, U., Knödlseder, J.: The COMPTEL $1.809 \mathrm{MeV}$ survey. In: Gimenez, A., Reglero, V., Winkler, C. (eds.) Exploring the Gamma-Ray Universe. ESA Special Publication, vol. 459, pp. 55-58 (2001)

Pöppel, W.G.L., Bajaja, E., Arnal, E.M., Morras, R.: The interstellar medium surrounding the Scorpius-Centaurus association revisited. Astron. Astrophys. 512, A83 (2010). https://doi.org/10. 1051/0004-6361/200811290

Prantzos, N.: Stellar nucleosynthesis and $\gamma$-ray line astronomy. Astron. Astrophys. Suppl. Ser. 120, C303 (1996)

Prantzos, N.: Origin of cosmic rays and evolution of spallogenic nuclides $\mathrm{Li}, \mathrm{Be}$ and B. In: Charbonnel, C., Tosi, M., Primas, F., Chiappini, C. (eds.) Light Elements in the Universe, vol. 268, pp. 473-482 (2010). https://doi.org/10.1017/ S1743921310004655. arXiv:1003.2317

Prantzos, N., Diehl, R.: Radioactive 26Al in the Galaxy: observations versus theory. Phys. Rep. 267, 1-69 (1996). https://doi.org/10. 1016/0370-1573(95)00055-0

Preibisch, T., Brown, A.G.A., Bridges, T., Guenther, E., Zinnecker, H.: Exploring the full stellar population of the Upper Scorpius OB association. Astron. J. 124, 404-416 (2002). https://doi.org/10. 1086/341174

Ramaty, R., Kozlovsky, B., Lingenfelter, R.E.: Nuclear gamma-rays from energetic particle interactions. Astrophys. J. Suppl. Ser. 40, 487-526 (1979). https://doi.org/10.1086/190596

Rodgers-Lee, D., Krause, M.G.H., Dale, J., Diehl, R.: Synthetic ${ }^{26} \mathrm{Al}$ emission from galactic-scale superbubble simulations. Mon. Not. R. Astron. Soc. 490(2), 1894-1912 (2019). https://doi.org/10. 1093/mnras/stz2708. arXiv:1909.10978

Ruiter, A.J., Belczynski, K., Sim, S.A., Hillebrandt, W., Fryer, C.L., Fink, M., Kromer, M.: Delay times and rates for Type Ia supernovae and thermonuclear explosions from double-detonation sub-Chandrasekhar mass models. Mon. Not. R. Astron. Soc. 417, 408-419 (2011). https://doi.org/10.1111/j.1365-2966.2011. 19276.x

Scalzo, R., Aldering, G., Antilogus, P., Aragon, C., Bailey, S., et al. (Nearby Supernova Factory): Type Ia supernova bolometric light curves and ejected mass estimates from the Nearby Supernova Factory. Mon. Not. R. Astron. Soc. 440(2), 1498-1518 (2014). https://doi.org/10.1093/mnras/stu350. arXiv:1402.6842

Schinnerer, E., Hughes, A., Leroy, A., Groves, B., Blanc, G.A., et al.: The gas-star formation cycle in nearby star-forming galaxies. I. Assessment of multi-scale variations. Astrophys. J. 887(1), 49 (2019). https://doi.org/10.3847/1538-4357/ab50c2. arXiv:1910. 10520 
Schulreich, M.M., Breitschwerdt, D., Feige, J., Dettbarn, C.: Numerical studies on the link between radioisotopic signatures on Earth and the formation of the Local Bubble. I. ${ }^{60} \mathrm{Fe}$ transport to the solar system by turbulent mixing of ejecta from nearby supernovae into a locally homogeneous interstellar medium. Astron. Astrophys. 604, A81 (2017). https://doi.org/10.1051/0004-6361/ 201629837. arXiv: 1704.08221

Seitenzahl, I.R., Townsley, D.M.: Nucleosynthesis in Thermonuclear Supernovae, p. 1955. Springer, Berlin (2017). https://doi.org/10. 1007/978-3-319-21846-5.87

Seitenzahl, I.R., Summa, A., Krauß, F., Sim, S.A., Diehl, R., et al.: $5.9-\mathrm{keV} \mathrm{Mn}$ K-shell X-ray luminosity from the decay of ${ }^{55} \mathrm{Fe}$ in Type Ia supernova models. Mon. Not. R. Astron. Soc. 447(2), 1484-1490 (2015). https://doi.org/10.1093/mnras/stu2537

Shafter, A.W.: The galactic nova rate revisited. Astrophys. J. 834(2), 196 (2017). https://doi.org/10.3847/1538-4357/834/2/196

Siegert, T.: Positron-annihilation spectroscopy through the Milky Way. $\mathrm{PhD}$ thesis, TU Munich (2017)

Sturner, S.J., Naya, J.E.: On the nature of the high-velocity ${ }^{26} \mathrm{Al}$ near the Galactic center. Astrophys. J. 526, 200-206 (1999). https:// doi.org/10.1086/307979

Summa, A., Elsässer, D., Mannheim, K.: Nuclear de-excitation line spectrum of Cassiopeia A. Astron. Astrophys. 533, A13 (2011). https://doi.org/10.1051/0004-6361/201117267

Summa, A., Ulyanov, A., Kromer, M., Boyer, S., Röpke, F.K., et al.: Gamma-ray diagnostics of Type Ia supernovae. Predictions of observables from three-dimensional modeling. Astron. Astrophys. 554, A67 (2013). https://doi.org/10.1051/0004-6361/201220972. arXiv: 1304.2777

Surman, R., McLaughlin, G.C., Hix, W.R.: Nucleosynthesis in the outflow from gamma-ray burst accretion disks. Astrophys. J. 643, 1057-1064 (2006). https://doi.org/10.1086/501116. arXiv:astro$\mathrm{ph} / 0509365$

Tatischeff, V., Gabici, S.: Particle acceleration by supernova shocks and spallogenic nucleosynthesis of light elements. Annu. Rev. Nucl. Part. Sci. 68(1), 377-404 (2018). https://doi.org/10.1146/annurevnucl-101917-021151. arXiv:1803.01794

Thielemann, F., Bitouzet, J., Kratz, K., Möller, P., Cowan, J.J., Truran, J.W.: Operation of the r-process and cosmochronology. Phys. Rep. 227, 269-281 (1993). https://doi.org/10.1016/03701573(93)90072-L

Thielemann, F.K., Wehmeyer, B., Wu, M.R.: r-Process sites, their ejecta composition, and their imprint in galactic chemical evolution. J. Phys. Conf. Ser. 1668, 012044 (2020). https://doi.org/10. 1088/1742-6596/1668/1/012044

Tibolla, O., Mannheim, K., Summa, A., Paravac, A., Greiner, J., Kanbach, G.: Nuclear lines revealing the injection of cosmic rays in supernova remnants. ArXiv e-prints (2011). arXiv:1106.1023

Vink, J.: Supernova remnants: the X-ray perspective. Astron. Astrophys. Rev. 20, 49 (2012). https://doi.org/10.1007/s00159-0110049-1. arXiv: 1112.0576

Vink, J., Kaastra, J.S., Bleeker, J.A.M., Bloemen, H.: The hard Xray emission and 44ti emission of Cas A. Adv. Space Res.
25, 689-694 (2000). https://doi.org/10.1016/S0273-1177(99) 00823-6

Voss, R., Diehl, R., Hartmann, D.H., Cerviño, M., Vink, J.S., Meynet, G., Limongi, M., Chieffi, A.: Using population synthesis of massive stars to study the interstellar medium near OB associations. Astron. Astrophys. 504, 531-542 (2009). https://doi.org/10.1051/ 0004-6361/200912260. arXiv:0907.5209

Voss, R., Diehl, R., Vink, J.S., Hartmann, D.H.: Probing the evolving massive star population in Orion with kinematic and radioactive tracers. Astron. Astrophys. 520, A51 (2010). https://doi.org/10. 1051/0004-6361/201014408. arXiv: 1005.3827

Wallner, A., Faestermann, T., Feige, J., Feldstein, C., Knie, K., et al.: Abundance of live ${ }^{244} \mathrm{Pu}$ in deep-sea reservoirs on Earth points to rarity of actinide nucleosynthesis. Nat. Commun. 6, 5956 (2015). https://doi.org/10.1038/ncomms6956. arXiv:1509.08054

Wallner, A., Feige, J., Kinoshita, N., Paul, M., Fifield, L.K., et al.: Recent near-Earth supernovae probed by global deposition of interstellar radioactive ${ }^{60} \mathrm{Fe}$. Nature 532, 69-72 (2016). https://doi.org/ 10.1038/nature17196

Wallner, A., Froehlich, M. B., Hotchkis, M. A. C., Kinoshita, N., Paul, M., Martschini, M., Pavetich, S., Tims, S. G., Kivel, N., Schumann, D., Honda, M., Matsuzaki, H., Yamagata, T., et al.: ${ }^{60} \mathrm{Fe}$ and ${ }^{244} \mathrm{Pu}$ deposited on Earth constrain the r-process yields of recent nearby supernovae. Science 6543, 742-745 (2021). https:// doi.org/10.1126/science.aax3972

Walsh, N., Binns, W., Israel, M., Murphy, R., Rauch, B.F., et al.: SuperTIGER abundances of galactic cosmic-rays for the charge interval $Z=41-56$. In: 36th International Cosmic Ray Conference (ICRC2019), International Cosmic Ray Conference, vol. 36, p. 147 (2019)

Wang, W., Lang, M.G., Diehl, R., Halloin, H., Jean, P., et al.: Spectral and intensity variations of Galactic ${ }^{26} \mathrm{Al}$ emission. Astron. Astrophys. 496, 713-724 (2009). https://doi.org/10.1051/0004-6361/ 200811175. arXiv:0902.0211

Wiedenbeck, M.E., Yanasak, N.E., Cummings, A.C., Davis Astron, J., George, J.S., et al.: The origin of primary cosmic rays: constraints from ACE elemental and isotopic composition observations. Space Sci. Rev. 99, 15-26 (2001). https://doi.org/10.1023/ A:1013816209004

Wu, M.R., Fernández, R., Martínez-Pinedo, G., Metzger, B.D.: Production of the entire range of r-process nuclides by black hole accretion disc outflows from neutron star mergers. Mon. Not. R. Astron. Soc. 463(3), 2323-2334 (2016). https://doi.org/10.1093/ mnras/stw2156. arXiv:1607.05290

Zinner, E.: Stellar nucleosynthesis and the isotopic composition of presolar grains from primitive meteorites. Annu. Rev. Earth Planet. Sci. 26, 147-188 (1998). https://doi.org/10.1146/annurev. earth.26.1.147

Zinner, E.: Stardust in the laboratory. Publ. Astron. Soc. Aust. 25, 7-17 (2008). https://doi.org/10.1071/AS07039 